\title{
Impact of N-Acetylcysteine on the Gut Microbiota in the Piglets Infected With Porcine Epidemic Diarrhea Virus
}

\author{
Tao $\mathrm{Wu}^{\dagger}$, Yang $\mathrm{Lyu}^{\dagger}$, Xueni Li, Mengjun Wu, Kui Yu, Siyuan Li, Changzheng Ji, Qian Zhang, \\ Yanyan Zhang, Di Zhao, Dan Yi and Yongqing Hou*
}

Hubei Key Laboratory of Animal Nutrition and Feed Science, Wuhan Polytechnic University (WPHU), Wuhan, China

This study was to investigate the impact of $\mathrm{N}$-acetylcysteine (NAC) on the gut microbiota in the healthy piglets and the piglets infected with porcine epidemic diarrhea virus (PEDV). Forty seven-day-old piglets were allocated into four groups: control group, NAC group (supplemented with $50 \mathrm{mg} / \mathrm{kg}$ body weight NAC), PEDV group (inoculated with $10^{4.5} \mathrm{TCID}_{50} \mathrm{PEDV}$ ), and PEDV+NAC group (PEDV infection +

\section{OPEN ACCESS}

Edited by:

Michael Kogut,

United States Department of Agriculture, United States

Reviewed by:

Jiangchao Zhao,

University of Arkansas, United States

Dirk Werling

Royal Veterinary College (RVC) United Kingdom

*Correspondence:

Yongqing $\mathrm{Hou}$

houyq@aliyun.com

tThese authors have contributed equally to this work and share first authorship

Specialty section:

This article was submitted to Veterinary Infectious Diseases,

a section of the journal

Frontiers in Veterinary Science

Received: 17 July 2020 Accepted: 11 December 2020 Published: 12 January 2021

Citation:

Wu T, Lyu Y, LiX, Wu M, YU K, Li S, Ji C, Zhang Q, Zhang Y, Zhao D, Yi D and Hou $Y$ (2021) Impact of

$N$-Acetylcysteine on the Gut

Microbiota in the Piglets Infected With

Porcine Epidemic Diarrhea Virus.

Front. Vet. Sci. 7:582338.

doi: 10.3389/fvets.2020.582338
NAC supplementation). The intestinal content was collected for DNA extraction and Illumina sequencing. The PEDV-infected piglets displayed distinct bacterial communities compared to the healthy piglets. PEDV infection decreased the abundance of Shigella and increased the abundance of Lactobacillus, Odoribacter, Anaerovibrio, Helicobacter, unclassified Lachnospiraceae, and Sutterella; affected several functions associated with metabolism, barrier, and immune. NAC supplementation decreased the abundance of unclassified Rikenellaceae and increased the abundance of Lactobacillus, Streptococcus, and Enterococcus in the healthy piglets, decreased the abundance of Oscillospira and Prevotella and increased the abundance of Lactobacillus in the PEDV-infected piglets; altered multiple functions involving in amino acid metabolism, cell signaling, cellular community, disease-related pathways, endocrine, and excretory system. In conclusion, PEDV infection caused severe dysbiosis of gut microbiome, whereas NAC supplementation played a positive role in regulating the gut microbiome during PEDV infection. Therefore, substances that can regulate gut microbiota could be ideal candidates to prevent or treat PEDV infection.

Keywords: $\mathrm{N}$-acetylcysteine, PEDV, dysbiosis, microbiome, piglets

\section{INTRODUCTION}

Great progress has been made to understand the role of gut microbiota in humans and animals alike (1). Biological functions of the gut microbiota to the host pertain to nutrition (2), metabolism (3), barrier, and immune (4), which are still expanding. Nevertheless, the relationship between gut microbiota and diseases remains unclear (5). For instance, increasing evidence have revealed that gut microbiota plays a vital role in the regulation, elimination, and potentiation of infectious diseases (6), while profound shifts of gut microbiota have frequently been reported in various diseases as well (7). Moreover, several enteric pathogens, such as Salmonella, Clostridium perfringens, and Escherichia coli, can also be found in healthy mammals (8). Therefore, more experiments are warranted to be established for a deeper understanding of microbiota alterations during diseases. 
Porcine epidemic diarrhea virus (PEDV) received increasing attention since the large-scale outbreaks in the last decades (9). PEDV is a devastating cause of diarrhea in pigs, which induces great economic losses to the pig industry worldwide (10). It causes severe diarrhea, vomiting, anorexia, dehydration, weight loss and high morbidity, and mortality in pigs of any age (11). Currently, research on PEDV mainly focused on molecular characterization, evolution, pathology, the host immune response and vaccinations, only limited knowledge has been expanded to the relationship between PEDV infection and porcine microbiota (12). Recent research has reported that PEDV infection induced dysbiosis of fecal and colonic microbiota in piglets and sows, leading to an increase in pathogenic bacteria and a decrease in commensal bacteria (13-17). However, only few studies focused on the microbiota alterations associated with the (expecting) anti-PEDV substances or compounds.

$\mathrm{N}$-acetylcysteine (NAC) is a source of sulfhydryl groups in cells and scavenger of free radicals as it interacts with reactive oxygen species such as hydroxide and hydrogen peroxide (18). NAC is rapidly metabolized by the small intestine to produce glutathione. Enterocytes, colonocytes, and other intestinal cells readily transport NAC and convert it into L-cysteine (19), which helps to maintain the integrity, growth, and function of the intestinal mucosa (20). Moreover, glutathione is a well-described and established antioxidant, it participates in detoxification of xenobiotics, regulation of cellular growth, modulation of immune response, and even inherent antibacterial properties (21), which is an important modulator of antibiotic activity in bacteria (22). This makes NAC a promising supplement for the piglets to regulate the porcine gut microbiome and alleviate dysbiosis during pathogenic infection (23), including PEDV (24). Our previous study also demonstrated that dietary NAC supplementation improved intestinal integrity and functions in PEDV-infected piglets (25). Based on the potential anti-PEDV effect of NAC in the piglets, this study was conducted to evaluate the impact of NAC on small intestinal microbiota in suckling piglets infected with PEDV. Findings of this study can contribute to understand the role of the gut microbiota during PEDV infection and provide vital clues for the treatment for PEDV infection.

\section{MATERIALS AND METHODS}

\section{Animals Experiments and Sample Collection}

The animal use protocol for this research was approved by the Animal Care and Use Committee of Wuhan Polytechnic University (1204-2016-0028). Forty seven-day-old crossbred (Duroc $\times$ Landrace $\times$ Large White) healthy piglets with 3.17 $\pm 0.25 \mathrm{~kg}$ initial body weight were purchased from a PEDVnegative farm. All piglets were randomly allocated into one of four treatment groups (Control, NAC, PEDV, PEDV+NAC; 10 replicates per group). All piglets were housed in clean pens with strict control of cross-infection. A commercial milk replacer (Wuhan Anyou Feed Co., Ltd.; Wuhan, China) formulated to meet the nutrition requirement for suckling piglets, was used as the basal diet during the trial. Prior to feeding, the milk replacer (powder) was dissolved in warm water $\left(45-55^{\circ} \mathrm{C}\right)$ to form a liquid feed (dry matter content of 20\%), as described by Wang et al. (25).

The entire trial period was 12 days. During day 5-9 of the trial, the piglets in the NAC and the PEDV + NAC groups were orally administered with $50 \mathrm{mg} / \mathrm{kg}$ body weight NAC (Sigma Chemical Inc., St. Louis, MO, USA; dissolved in the liquid milk replacer), and the piglets in the other two groups received the same volume of the liquid milk replacer. On day 9 of the trial, PEDV at a dose of $10^{4.5}$ TCID $_{50}(50 \%$ tissue culture infectious dose) per piglet was orally inoculated to the piglets in the PEDV and the PEDV + NAC groups, the same volume of sterile saline was inoculated to the piglets in control and NAC groups. On day 12 , all piglets were sacrificed to obtain the intestinal content from jejunum, ileum, colon, and cecum. All samples were rapidly frozen in liquid nitrogen and then stored at $-80^{\circ} \mathrm{C}$ until analysis.

\section{DNA Extraction}

The bacterial DNA was extracted from the intestinal content using the QIAampDNA Stool Mini Kit (Qiagen, Hilden, Germany) according to the manufacturer's protocol. The extracted DNA was purified by using the QIAamp spin column (Qiagen, Hilden, Germany). Total DNA was quantified by determining the OD $260 \mathrm{~nm} / \mathrm{OD} 280 \mathrm{~nm}$ ratio using the NanoDrop ${ }^{\circledR}$ ND-1000A UV-VIS spectrophotometer (Thermo Scientific, Wilmington, DE, USA). The quality of microbial DNA in each sample was evaluated by $1 \%$ denatured agarose gel electrophoresis and the quantity of DNA with Picogreen assay. The microbial DNA were normalized with $10 \mathrm{mM}$ Tris buffer $(\mathrm{pH}$ $8.5)$ to $5 \mathrm{ng} / \mathrm{ul}$.

\section{Amplification and Sequencing}

The V3-V4 region of the 16S rRNA gene was amplified by two stage polymerase chain reaction (PCR). The first stage of PCR was processed using the HiFi HotStart ReadyMix kit (Kapa Biosystems, Wilmington, MA, USA) with the forward primer: 5'-TCGTCGGCAGCG TCAGATGTGTATAAGAGACAGCCTACGGGNGGCWGCAG $-3^{\prime}$ and the reverse primer $5^{\prime}$-GTCTCGTGGGCTCGGAGATG TGTATAAGAGACAGGACTACHVGGGTATCT AATCC-3'. The total reaction volume of PCR was $25 \mu \mathrm{L}$, containing $12.5 \mu \mathrm{L}$ of $2 \times$ HotStart ReadyMix Buffer, $0.5 \mu \mathrm{L}$ of each primer $(5 \mathrm{mM})$ and $10 \mathrm{ng}$ of template DNA. PCR thermocycling conditions were denaturation at $95^{\circ} \mathrm{C}$ for $3 \mathrm{~min}(1 \mathrm{cycle}), 95^{\circ} \mathrm{C}$ for $30 \mathrm{~s}$, annealing at $55^{\circ} \mathrm{C}$ for $30 \mathrm{~s}$, elongation at $72^{\circ} \mathrm{C}$ for $30 \mathrm{~s}$ and a final extension at $72^{\circ} \mathrm{C}$ for $5 \mathrm{~min}$. The second stage was index PCR. This step attaches dual indices and Illumina sequencing adapters using the Nextera XT Index Kit (Illumina, San Diego, CA, USA). The total reaction volume was $50 \mu \mathrm{L}$, containing $25 \mu \mathrm{L}$ of $2 \times$ HotStart ReadyMix Buffer, $5 \mu \mathrm{L}$ Cleaned PCR product, $5 \mu \mathrm{L}$ N7 index and $5 \mu \mathrm{L}$ S5 index primer. PCR thermocycling conditions were $95^{\circ} \mathrm{C}$ for $3 \mathrm{~min}(1 \mathrm{cycle}), 95^{\circ} \mathrm{C}$ for $30 \mathrm{~s}$, annealing at $55^{\circ} \mathrm{C}$ for $30 \mathrm{~s}$, elongation at $72^{\circ} \mathrm{C}$ for $30 \mathrm{~s}$ ( 8 cycles), and a final extension at $72^{\circ} \mathrm{C}$ for $5 \mathrm{~min}$. The PCR production was purified with the Agencourt AMPure XP purification system (Beckman, Brea, CA, USA) and the concentration was measured with SpectraMax i3X Multi-mode detection platform (Molecular Devices, San Jose, 
TABLE 1 | Alpha diversity of gut microbiota in piglets.

\begin{tabular}{|c|c|c|c|c|c|}
\hline Item & Control & NAC & PEDV & PEDV+NAC & $p$-value \\
\hline \multicolumn{6}{|l|}{ Jejunum } \\
\hline Shannon & $1.76 \pm 0.66^{b}$ & $1.76 \pm 0.74^{b}$ & $2.32 \pm 0.28^{a b}$ & $2.72 \pm 0.78^{a}$ & 0.008 \\
\hline Simpson & $0.60 \pm 0.21^{b}$ & $0.59 \pm 0.24^{b}$ & $0.80 \pm 0.04^{a}$ & $0.83 \pm 0.14^{a}$ & 0.006 \\
\hline Chao1 & $91.98 \pm 5.65$ & $96.19 \pm 2.79$ & $94.74 \pm 2.76$ & $92.22 \pm 4.77$ & 0.134 \\
\hline \multicolumn{6}{|l|}{ Ileum } \\
\hline Shannon & $1.80 \pm 0.74$ & $1.98 \pm 0.57$ & $2.11 \pm 0.72$ & $2.30 \pm 0.58$ & 0.403 \\
\hline Simpson & $0.56 \pm 0.22$ & $0.63 \pm 0.17$ & $0.65 \pm 0.21$ & $0.73 \pm 0.16$ & 0.329 \\
\hline Chao1 & $102.57 \pm 4.57^{b}$ & $106.68 \pm 2.04^{a}$ & $105.37 \pm 4.02^{a b}$ & $105.19 \pm 4.69^{a b}$ & 0.170 \\
\hline \multicolumn{6}{|l|}{ Colon } \\
\hline Shannon & $3.40 \pm 0.19$ & $3.09 \pm 0.41$ & $3.34 \pm 0.37$ & $3.18 \pm 0.42$ & 0.257 \\
\hline Simpson & $0.92 \pm 0.02$ & $0.89 \pm 0.04$ & $0.92 \pm 0.03$ & $0.91 \pm 0.04$ & 0.207 \\
\hline Chao1 & $166.09 \pm 10.57$ & $159.63 \pm 16.28$ & $156.78 \pm 25.73$ & $157.53 \pm 26.75$ & 0.777 \\
\hline \multicolumn{6}{|l|}{ Cecum } \\
\hline Shannon & $3.23 \pm 0.23$ & $3.10 \pm 0.43$ & $3.22 \pm 0.40$ & $3.16 \pm 0.33$ & 0.850 \\
\hline Simpson & $0.90 \pm 0.03$ & $0.88 \pm 0.05$ & $0.91 \pm 0.04$ & $0.91 \pm 0.02$ & 0.340 \\
\hline Chao1 & $162.66 \pm 11.78$ & $162.71 \pm 12.96$ & $161.02 \pm 17.26$ & $148.52 \pm 22.34$ & 0.214 \\
\hline
\end{tabular}

${ }^{a, b}$ Values with different letters are significantly different $(p<0.05)$.

CA, USA). The purified DNA samples were sequenced using Illumina MiSeq platform (San Diego, CA, USA).

\section{Data Processing and Statistical Analysis}

The raw reads were put for quality control after base calling, and then were subjected to pipeline QIIME for OTU picking and taxonomy as described (26). Alpha diversity (Shannon, Simpson, and Chao1) were calculated, and the one-way analysis of variance (ANOVA) with a Turkey's test as post-hoc was used to compare between the groups. The beta diversity (Bray-Curtis Index) was calculated using principal coordinate analysis ( $\mathrm{PCoA}$ ), with further comparison of groups by analysis of similarities (ANOSIM). Dendrograms and colinear relation diagrams were generated using Majorbio Cloud Platform (Shanghai Majorbio Bio-pharm Technology, Shanghai, China). Relative abundance of operational taxonomic units (OTUs) were compared by one-way analysis of variance (ANOVA) using Majorbio Cloud Platform, with a Turkey's test as post-hoc and p-values adjusted by false discovery rate (FDR). Functional potentials of OTUs were evaluated by KEGG (Kyoto Encyclopedia of Genes and Genomes) pathway enrichment using PICRUSt (http:// huttenhower.sph.harvard.edu/galaxy/), a heatmap was generated using TBtools (https://github.com/CJ-Chen/TBtools), and the comparison was processed by ANOVA using Majorbio Cloud Platform with a Turkey's test as post-hoc. Significance was set at $p$ $<0.05$ for all comparisons.

\section{RESULTS}

\section{Sequencing Data and OUT Clustering}

A total of 3,629,889 reads were obtained with an average length of 460.36 bp across all samples, with 3,629,889 reads from jejunum, 4,658,260 reads from ileum, 2,987,358 reads from colon, and 2,936,753 reads from cecum. The good coverage was obtained over $99.9 \%$ for all samples, reflecting reliable accuracy of the sequencing.

Evenness, diversity, and richness were calculated by Shannon, Simpson, and Chaol indices, respectively (Table 1). Compared to the control group, the NAC group increased the Chaol index in the ileum, the PEDV group increased the Shannon index in the jejunum, and the PEDV and the PEDV+NAC group increased the Simpson index in the jejunum $(p<0.05)$. No significant difference was observed in the colon and cecum.

Beta diversity was calculated by PCoA based on the BrayCurtis index. There is no clear clustering in the ileum $(p=0.398)$ (Figure 1B), while distinct bacterial communities were observed in the jejunum $(p=0.002)$ (Figure 1A), colon $(p<0.001)$ (Figure 1C), and cecum $(p<0.001)$ (Figure 1D). Particularly, the result illustrated obvious clusters between the control and the NAC groups as well as between the PEDV and the PEDV+NAC groups in the jejunum, colon, and cecum.

\section{Structure of Intestinal Microbiota}

The majority of microbiota in all samples was composed of four predominant bacterial phyla: Proteobacteria, Firmicutes, Bacteroidetes, and Fusobacteria, accounting for an average of over $96 \%$ of total sequences (Figure 2). In the jejunum, the proportions of the four phyla in the control group were 72.5 , 23.5, 2.2, and 1.2\%; that in the NAC group were 59.4, 37.7, 2.1 , and $0.4 \%$; that in the PEDV group were 20.9, 75.0, 3.0, and $0.6 \%$; that in the PEDV+NAC group were 18.7, 69.6, 9.4, and $1.2 \%$. In the ileum, the proportions of the four phyla were 83.0, 12.9, 3.3, and $0.5 \%$ in the control group; 68.1, 24.9, 5.7, and $0.5 \%$ in the NAC group; $74.3,15.8,8.6$, and $0.6 \%$ in the PEDV group; 59.1, 32.9, 6.4, and $0.6 \%$ in the PEDV+NAC group. In the colon, the proportions were 6.7, 24.1, 63.6, and $0.1 \%$ in the control group; 6.2, 25.0, 61.8, and $0.1 \%$ in the NAC group; 15.0, 38.4, 38.7, and $2.0 \%$ in the PEDV group, 14.9, 36.9, 


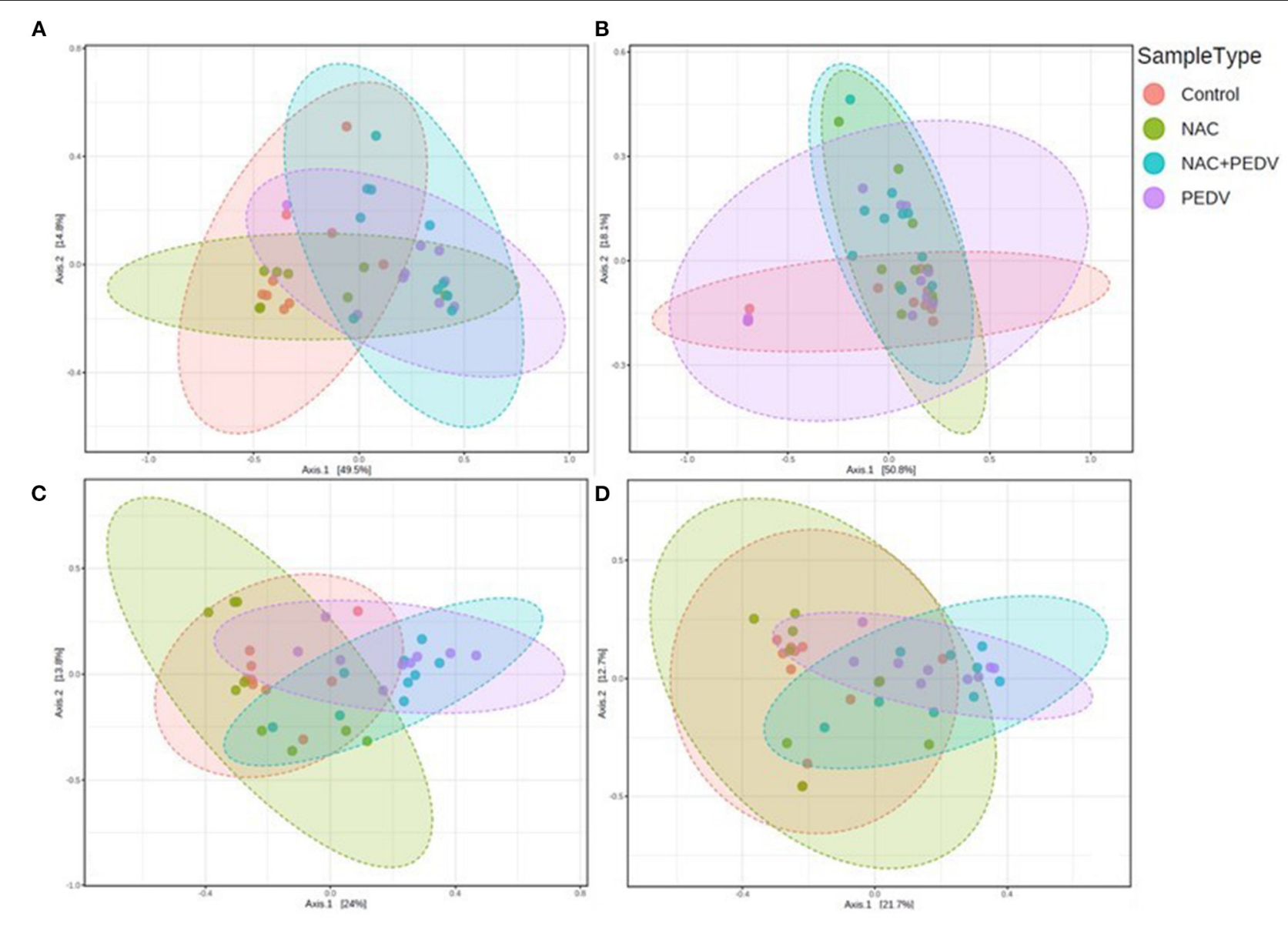

FIGURE 1 | Beta diversity of gut microbiota in piglets. (A) Jejunum, $p=0.002$; (B) ileum, $p=0.398$; (C) colon, $p<0.001$; (D) cecum, $p<0.001$.

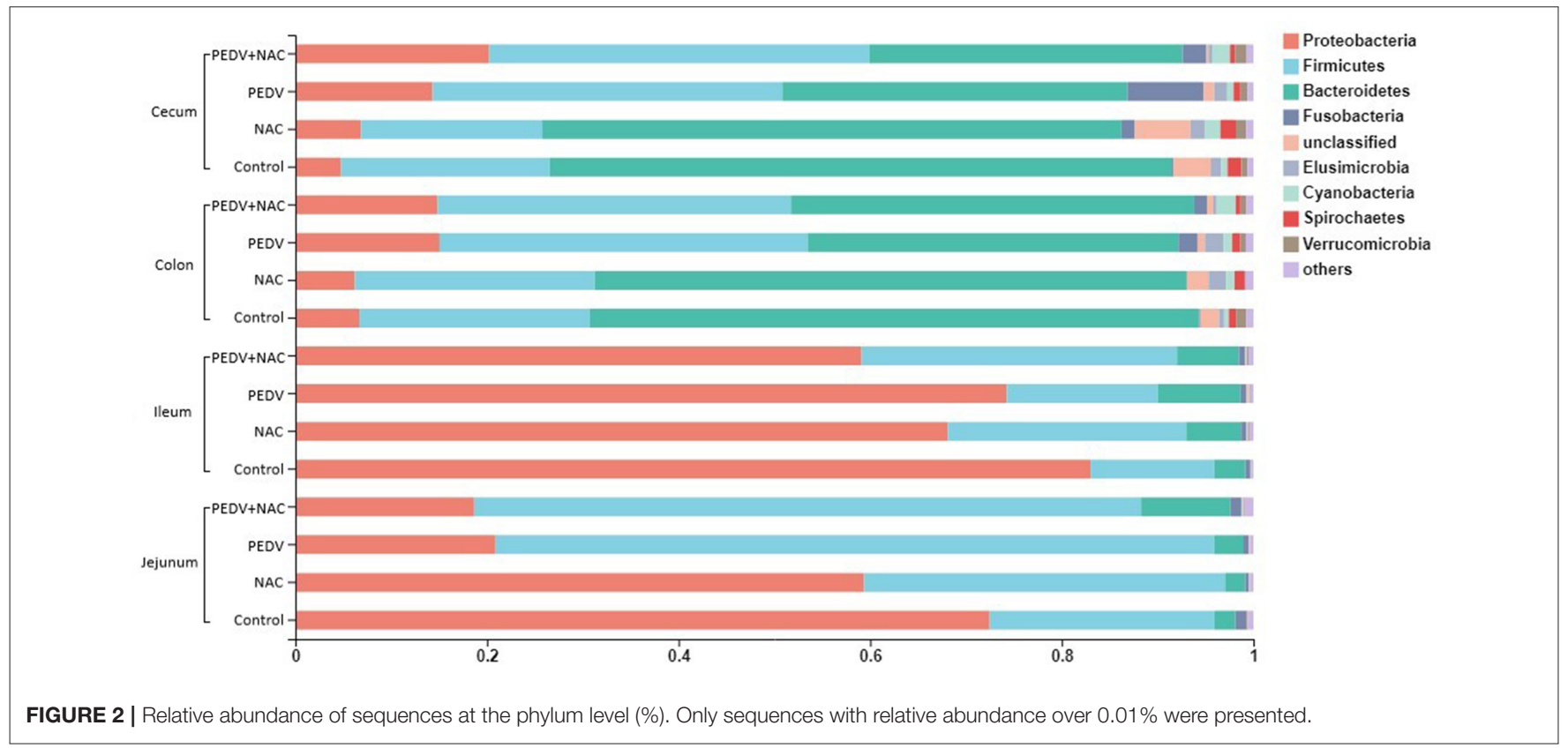


A

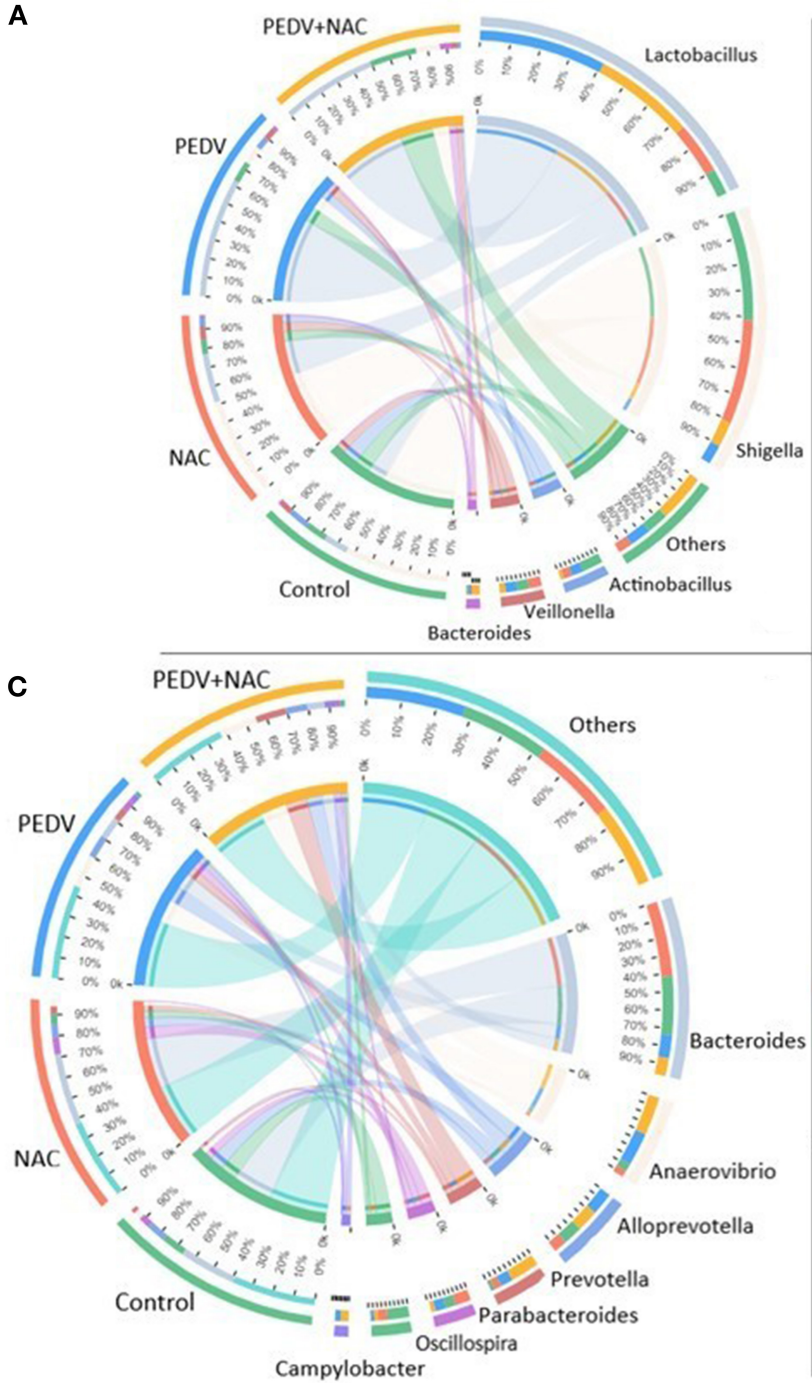

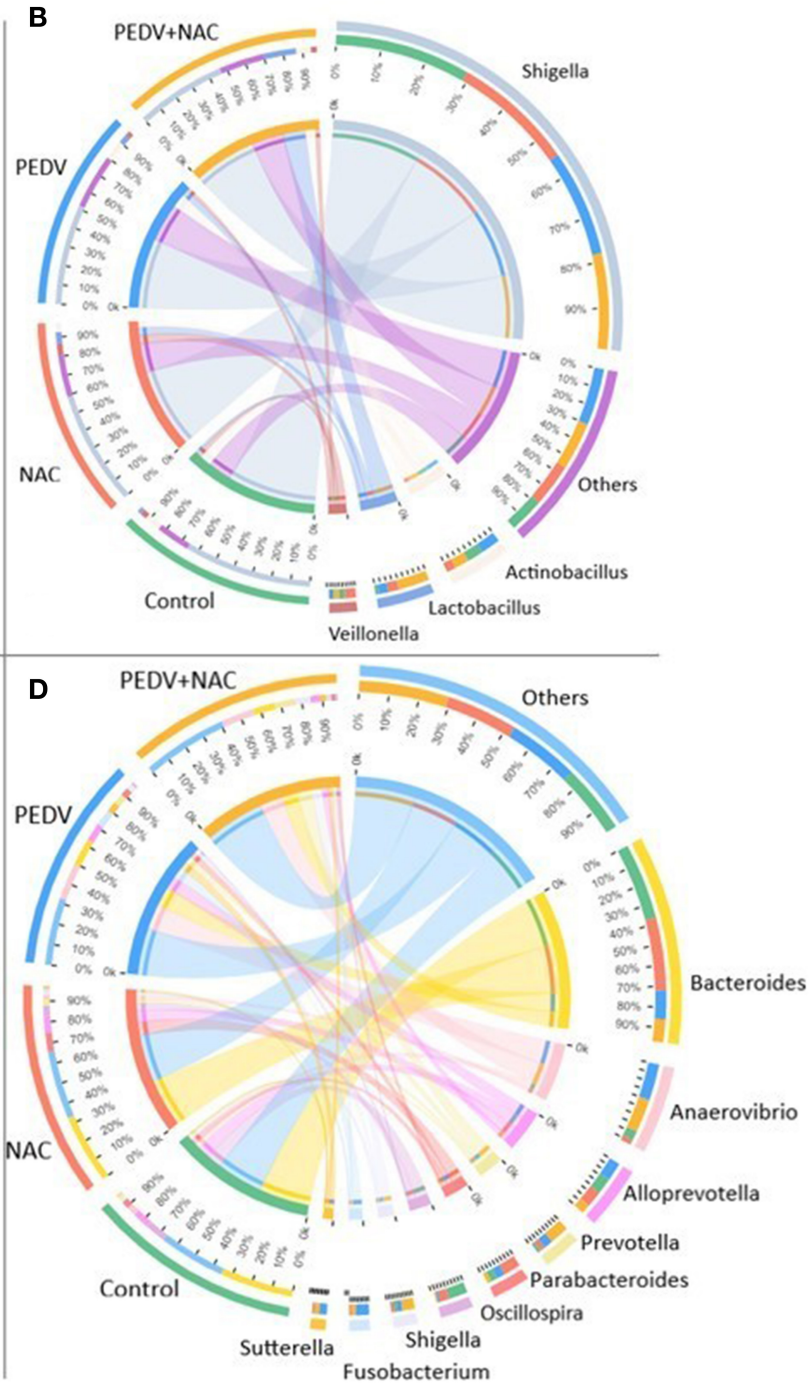

FIGURE 3 | Relative abundance of OTUs at the genus level (\%). (A) Jejunum; (B) ileum; (C) colon; (D) cecum. Only OTUs with relative abundance over 2\% were presented.

42.1, and $1.3 \%$ in the PEDV+NAC group. In the cecum, the proportions were $4.8,21.7,65.0$, and $0.2 \%$ in the control group; $6.9,18.9,60.4$, and $1.4 \%$ in the NAC group; 14.3, 36.4, 36.0, and $8.0 \%$ in the PEDV group, 20.2, 39.7, 32.7, and $2.5 \%$ in the $\mathrm{PEDV}+\mathrm{NAC}$ group.

A total of 13 genera was identified as the dominant bacterial populations ( $>2 \%$ ) in all groups. Shigella, Lactobacillus, Actinobacillus and Veillonella were the most abundant genera in the jejunum, accounting for $53,13,12$, and $6.4 \%$ in the control group, 52, 27, 5.1, and 7.1\% in the NAC group, 63, 9, 5.7, and $6.1 \%$ in the PEDV group, 51, 13, 1.6, and $3.3 \%$ in the PEDV+NAC group (Figure 3A). Shigella, Actinobacillus, Lactobacillus, and Veillonella were the most abundant genera in the ileum, accounting for $63,7.9,1.9$, and $3.3 \%$ in the control group, 59, 5.4, 5.9, and $6.4 \%$ in the NAC group, 48 , 8.7, 4.4, and $2.4 \%$ in the PEDV group, 46, 8.1, 17, and $3.0 \%$ in the PEDV+NAC group (Figure 3B). Bacteroides, Anaerovibrio, Alloprevotella, Prevotella, Parabacteriodies, and Oscillospria were the most abundant genera in the colon, accounting for 27, 3.8, $9.5,1.9,5.4$, and $11 \%$ in the control group, $35,3.3,6.4,3.8,8.1$, and $4.8 \%$ in the NAC group, $11,16,11,6.1,5.3$, and $1.7 \%$ in the PEDV group, $8.5,19,11,15,2.3$, and $2.2 \%$ in the PEDV+NAC group (Figure 3C). Bacteroides, Anaerovibrio, Alloprevotella, Prevotella, Parabacteriodies, and Oscillospria were the most abundant genera in the cecum, accounting for $36,4.6,8.3,1.7,3.7$, and $10 \%$ in the control group, $34,2.5,8.3,2.9,9.3$, and $5 \%$ in the NAC group, 14 , $18,9.1,5,4.3$, and $1.3 \%$ in the PEDV group, 11, 16, 4.8, 10, 2.1, and $0.1 \%$ in the PEDV+NAC group (Figure $3 D$ ). 
TABLE 2 | Comparison of OUT abundance (\%).

\begin{tabular}{|c|c|c|c|c|c|}
\hline Taxonomy & Control & NAC & PEDV & PEDV+NAC & $p$ \\
\hline \multicolumn{6}{|l|}{ Jejunum } \\
\hline o_Enterobacteriales; f_Enterobacteriaceae; g_Shigella & $36.43 \pm 22.29^{a}$ & $36.93 \pm 20.91^{a}$ & $7.44 \pm 9.79^{b}$ & $7.57 \pm 9.23^{b}$ & 0.038 \\
\hline o_Enterobacteriales; f_Enterobacteriaceae; g_Shigella & $14.94 \pm 9.25^{\mathrm{a}}$ & $16.70 \pm 9.91^{\mathrm{a}}$ & $3.71 \pm 4.71^{b}$ & $3.18 \pm 3.99^{b}$ & 0.038 \\
\hline o_Lactobacillales; f_Lactobacillaceae; g_Lactobacillus & $0.10 \pm 0.09^{b}$ & $0.90 \pm 1.42^{b}$ & $5.31 \pm 3.27^{a}$ & $4.48 \pm 5.29^{a}$ & 0.067 \\
\hline o_Clostridiales; f_Lachnospiraceae; g_ & $0.33 \pm 0.42^{a}$ & $0.11 \pm 0.09^{a b}$ & $0.05 \pm 0.06^{b}$ & $0.01 \pm 0.01^{b}$ & 0.067 \\
\hline o_Lactobacillales; f_Lactobacillaceae; g_Lactobacillus & $0.02 \pm 0.03^{b}$ & $0.06 \pm 0.07^{b}$ & $0.14 \pm 0.08^{a}$ & $0.15 \pm 0.12^{\mathrm{a}}$ & 0.067 \\
\hline \multicolumn{6}{|l|}{ Ileum } \\
\hline o_Lactobacillales; f_Lactobacillaceae; g_Lactobacillus & $0.40 \pm 0.47^{c}$ & $2.63 \pm 2.53^{b}$ & $1.53 \pm 1.78^{\mathrm{bc}}$ & $8.32 \pm 9.49^{a}$ & 0.045 \\
\hline o_Lactobacillales; f_Streptococcaceae; g_Streptococcus & $0.06 \pm 0.07^{b}$ & $0.15 \pm 0.10^{\mathrm{a}}$ & $0.04 \pm 0.02^{b}$ & $0.11 \pm 0.11^{\mathrm{ab}}$ & 0.096 \\
\hline o_Bacteroidales; f_Paraprevotellaceae; g_Prevotella & $0.02 \pm 0.04^{b}$ & $0.01 \pm 0.03^{b}$ & $0.08 \pm 0.09^{a}$ & $0.01 \pm 0.01^{b}$ & 0.096 \\
\hline o_Lactobacillales; f_Enterococcaceae; g_Enterococcus & $0.01 \pm 0.02^{b}$ & $0.06 \pm 0.07^{a}$ & $0.01 \pm 0.01^{b}$ & $0.01 \pm 0.01^{\mathrm{b}}$ & 0.096 \\
\hline o_Bacteroidales; f_Odoribacteraceae; g_Odoribacter & $0.01 \pm 0.01^{b}$ & $0.02 \pm 0.03^{b}$ & $0.05 \pm 0.05^{a}$ & $0.03 \pm 0.03^{\mathrm{ab}}$ & 0.096 \\
\hline \multicolumn{6}{|l|}{ Colon } \\
\hline o_Selenomonadales; f_Selenomonadaceae; g_Anaerovibrio & $2.75 \pm 4.07^{\mathrm{b}}$ & $1.40 \pm 1.98^{b}$ & $7.65 \pm 5.76^{\mathrm{ab}}$ & $9.78 \pm 6.09^{a}$ & 0.034 \\
\hline o_Selenomonadales; f_Selenomonadaceae; g_Anaerovibrio & $1.89 \pm 2.91^{\mathrm{b}}$ & $0.83 \pm 1.17^{b}$ & $5.19 \pm 3.72^{a}$ & $5.83 \pm 3.47^{a}$ & 0.034 \\
\hline o_Selenomonadales; f_Selenomonadaceae; g_Anaerovibrio & $1.35 \pm 2.04^{b}$ & $0.62 \pm 0.87^{b}$ & $3.83 \pm 2.82^{\mathrm{a}}$ & $4.48 \pm 2.75^{\mathrm{a}}$ & 0.083 \\
\hline o_Lachnospirales; f_Lachnospiraceae; g_ & $0.45 \pm 0.67^{b}$ & $0.08 \pm 0.08^{b}$ & $3.81 \pm 3.70^{\mathrm{a}}$ & $2.05 \pm 2.72^{\mathrm{ab}}$ & 0.083 \\
\hline o_Lachnospirales; f_Lachnospiraceae; g_Lachnoclostridium & $0.23 \pm 0.22^{b}$ & $0.44 \pm 0.39^{b}$ & $2.08 \pm 1.67^{a}$ & $1.50 \pm 1.17^{\mathrm{ab}}$ & 0.083 \\
\hline o_Campylobacterales; f_Helicobacteraceae; g_Helicobacter & $0.01 \pm 0.01^{\mathrm{b}}$ & $0.04 \pm 0.11^{b}$ & $1.31 \pm 0.95^{\mathrm{a}}$ & $0.63 \pm 1.12^{\mathrm{ab}}$ & 0.083 \\
\hline o_Acidaminococcales; f_Acidaminococcaceae; g_Acidaminococcus & $0.04 \pm 0.09^{b}$ & $1.22 \pm 1.56^{\mathrm{a}}$ & $0.01 \pm 0.02^{b}$ & $0.12 \pm 0.32^{b}$ & 0.167 \\
\hline o_Burkholderiales; f_Sutterellaceae; g_Sutterella & $0.15 \pm 0.17^{b}$ & $0.05 \pm 0.06^{b}$ & $0.46 \pm 0.51^{a}$ & $0.31 \pm 0.36^{\mathrm{a}}$ & 0.167 \\
\hline o_Oscillospirales; f_Oscillospiraceae; g_ & $0.05 \pm 0.11^{b}$ & $0.08 \pm 0.17^{b}$ & $0.32 \pm 0.25^{\mathrm{ab}}$ & $0.55 \pm 0.61^{\mathrm{a}}$ & 0.167 \\
\hline o_Lactobacillales; f_Lactobacillaceae; g_Lactobacillus & $0.06 \pm 0.09^{b}$ & $0.02 \pm 0.04^{b}$ & $0.15 \pm 0.22^{\mathrm{ab}}$ & $0.69 \pm 0.75^{a}$ & 0.167 \\
\hline o_Veillonellales; f_Veillonellaceae; g_Allisonella & $0.15 \pm 0.41^{b}$ & $0.06 \pm 0.12^{b}$ & $0.02 \pm 0.03^{b}$ & $0.77 \pm 1.03^{\mathrm{a}}$ & 0.167 \\
\hline o_Lachnospirales; f_Lachnospiraceae; g_Lachnoclostridium & $0.05 \pm 0.05^{b}$ & $0.10 \pm 0.07^{b}$ & $0.51 \pm 0.39^{a}$ & $0.35 \pm 0.26^{\mathrm{ab}}$ & 0.167 \\
\hline o_Bacteroidales; f_Rikenellaceae; g_ & $0.43 \pm 0.45^{a}$ & $0.12 \pm 0.14^{b}$ & $0.02 \pm 0.02^{b}$ & $0.03 \pm 0.05^{b}$ & 0.167 \\
\hline \multicolumn{6}{|l|}{ Cecum } \\
\hline o_Selenomonadales; f_Selenomonadaceae; g_Anaerovibrio & $2.88 \pm 4.38^{b}$ & $3.08 \pm 4.57^{b}$ & $9.60 \pm 7.13^{a}$ & $6.78 \pm 5.35^{\mathrm{ab}}$ & 0.070 \\
\hline o_Selenomonadales; f_Selenomonadaceae; g_Anaerovibrio & $1.60 \pm 3.10^{\mathrm{b}}$ & $0.85 \pm 0.74^{b}$ & $6.86 \pm 4.17^{\mathrm{a}}$ & $4.53 \pm 2.91^{\mathrm{a}}$ & 0.070 \\
\hline o_Clostridiales; f_Ruminococcaceae; g_Oscillospira & $0.85 \pm 0.72^{b}$ & $0.70 \pm 0.54^{b}$ & $2.97 \pm 2.79^{a}$ & $1.22 \pm 1.41^{\mathrm{ab}}$ & 0.070 \\
\hline o_Clostridiales; f_Lachnospiraceae; g_ & $0.19 \pm 0.21^{b}$ & $0.60 \pm 0.51^{b}$ & $1.97 \pm 1.69^{a}$ & $1.55 \pm 1.45^{\mathrm{a}}$ & 0.124 \\
\hline o_Bacteroidales; f_Prevotellaceae; g_Prevotella & $1.23 \pm 2.35^{\mathrm{a}}$ & $1.44 \pm 1.65^{\mathrm{a}}$ & $0.02 \pm 0.02^{b}$ & $0.02 \pm 0.01^{b}$ & 0.124 \\
\hline 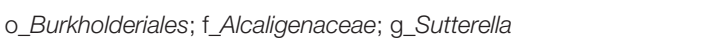 & $0.15 \pm 0.26^{b}$ & $0.21 \pm 0.25^{b}$ & $0.97 \pm 0.93^{\mathrm{a}}$ & $0.47 \pm 0.56^{\mathrm{ab}}$ & 0.124 \\
\hline o_Clostridiales; f_Lachnospiraceae; g_ & $0.05 \pm 0.05^{b}$ & $0.13 \pm 0.12^{\mathrm{ab}}$ & $0.30 \pm 0.35^{a}$ & $0.16 \pm 0.12^{a b}$ & 0.124 \\
\hline o_Bacteroidales; f_Odoribacteraceae; g_Butyricimonas & $0.09 \pm 0.10^{b}$ & $0.13 \pm 0.12^{\mathrm{ab}}$ & $0.30 \pm 0.35^{a}$ & $0.16 \pm 0.12^{\mathrm{ab}}$ & 0.124 \\
\hline o_Clostridiales; f_Ruminococcaceae; g_Oscillospira & $0.20 \pm 0.17^{a}$ & $0.12 \pm 0.20^{\mathrm{ab}}$ & $0.25 \pm 0.18^{a}$ & $0.08 \pm 0.05^{b}$ & 0.124 \\
\hline
\end{tabular}

${ }^{a, b, c}$ Values with different letters are significantly different $(p<0.05)$.

f_Lachnospiraceae; g_means an unclassified genus that belongs to family Lachnospiraceae, same as others.

\section{Comparison of OTUs Abundance}

The differences in the percentage of OTU abundance between all groups were summarized in Table 2. In the jejunum, compared to the control group, both the PEDV and the PEDV+NAC groups decreased the abundance of Shigella and Lachnospiraceae_g (an unclassified genus that belongs to family Lachnospiraceae, same as others), increased the abundance of Lactobacillus ( $p$ $<0.05$ ). No significant difference was observed between the control and the NAC groups as well as between the PEDV and the PEDV+NAC groups. In the ileum, compared to the control group, the NAC group increased the abundance of Lactobacillus, Streptococcus and Enterococcus, the PEDV group increased the abundance of Prevotella and Odoribacter, and the PEDV+NAC group increased the abundance of Lactobacillus $(p<0.05)$; compared to the PEDV group, the PEDV+NAC group decreased the abundance of Prevotella and increased the abundance of Lactobacillus $(p<0.05)$. In the colon, compared to the control group, the NAC group decreased the abundance of Rikenellaceae_g and increased the abundance of Acidaminococcus, the PEDV group decreased the abundance of Rikenellaceae_g and increased the abundance of Anaerovibrio, Lachnospiraceae_g, Lachnoclostridium, Helicobacter, and Sutterella $(p<0.05)$; compared to the PEDV group, the PEDV + NAC group increased the abundance of Allisonella $(p<$ 


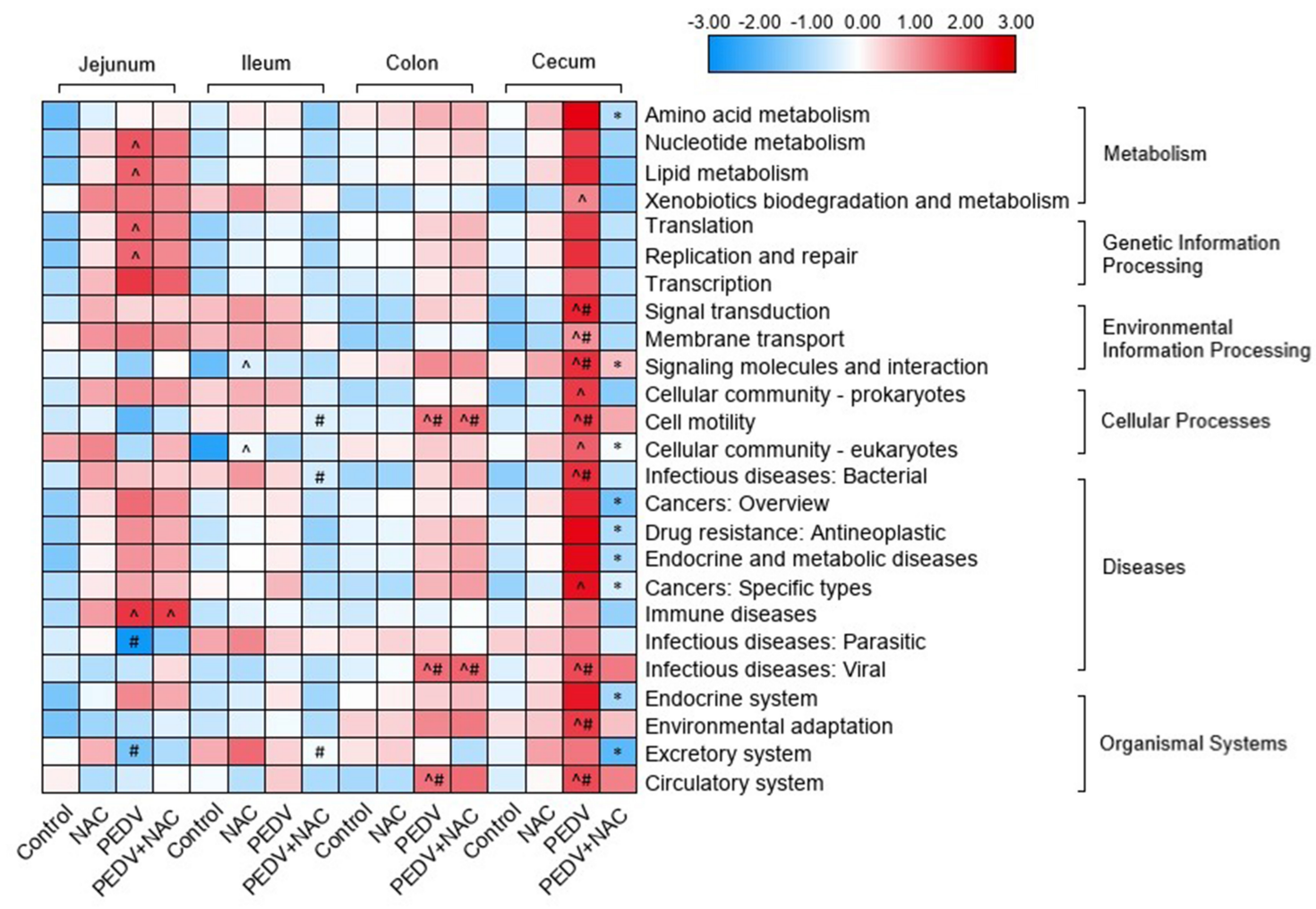

FIGURE 4 | KEGG pathway enrichment of gut microbiome in piglets. Different colors represent the values of log-transformed abundances of KEGG pathways. ${ }^{\wedge}$ means significant difference compared to the control group $(p<0.05)$; \# means significant difference compared to the NAC group ( $\left.p<0.05\right)$; ${ }^{*}$ means significant difference compared to the PEDV group ( $p<0.05)$.

0.05). In the cecum, compared to the control group, the PEDV group decreased the abundance of Prevotella and increased the abundance of Anaerovibrio, Oscillospira, Lachnospiraceae_g, Helicobacter, Sutterella, and Butyricimonas $(p<0.05)$; compared to the PEDV group, the PEDV+NAC group decreased the abundance of Oscillospira $(p<0.05)$.

\section{Functional Potentials of OUTs}

Functional potentials of the microbiome profile were evaluated by KEGG pathway enrichment, the relative abundance of 25 pathways was found with significant differences between groups (Figure 4). In the jejunum, compared to the control group, the PEDV group increased the abundance of pathways "nucleotide metabolism," "lipid metabolism," "translation," "replication and repair," "transcription," and "immune diseases," the PEDV+NAC group increased the abundance of pathway "immune diseases" ( $p$ $<0.05$ ); compared to the NAC group, the PEDV group increased the abundance of pathway "infectious diseases: parasitic" and decreased the abundance of pathway "excretory system" $(p<$ 0.05). In the ileum, compared to the control group, the NAC group increased the abundance of pathways "signaling molecules and interaction" and "cellular community-eukaryotes" $(p<$ $0.05)$; compared to the NAC group, the PEDV+NAC group decreased the abundance of pathways "cell motility," "infectious diseases: bacterial" and "excretory system" $(p<0.05)$. In the colon, compared to the control and the NAC groups, the PEDV and the PEDV+NAC groups increased the abundance of pathways "cell motility," "infectious diseases: viral," and "circulatory system" ( $p<0.05)$. In the cecum, compared to the control group, the PEDV group also increased the abundance of pathways "xenobiotics biodegradation and metabolism," "cellular community-prokaryotes," "cellular community-eukaryotes," and "cancers: specific types" ( $p<0.05)$; compared to the control and the NAC groups, the PEDV group increased the abundance of pathways "signal transduction," "membrane transport," "signaling molecules and interaction," "cell motility," "infectious diseases: bacterial," "infectious diseases: viral," "environmental adaptation," and "circulatory system" ( $p<0.05)$; compared the PEDV group, the PEDV+NAC group decreased the abundance of pathways "amino acid metabolism," "signaling molecules and interaction," "cellular community-eukaryotes," "canceroverview," "drug resistance-antineoplastic," "endocrine and metabolic diseases," "cancers: specific types," "endocrine system", and "excretory system" $(p<0.05)$.

\section{DISCUSSION}

Several studies have revealed the dysbiosis of porcine gut microbiome induced by PEDV infection (13-17), which 
was associated with the increased abundance of pathogenic bacteria (e.g., Shigella, Enterococcus, and Fusobacterium) and the decreased abundance of short-chain fatty acid (SCFA)producing bacteria (e.g., Bacteroides, Prevotella, Butyricimonas, and Alistipes). However, for the first time this study investigated the effect of a potential anti-PEDV substance on the gut microbiota in piglets, NAC was supplemented to the diet of both the healthy and PEDV-infected piglets. Our previous study indicated that NAC improved mucosal structure and intestinal permeability, reduced oxidative stress, and modulated the expression of genes associated with intestinal immune in the piglets during PEDV infection (25). Therefore, we hypothesize that the supplementation of NAC is promising to protect piglets from the microbiome dysbiosis caused by PEDV infection.

In the present study, the ileal samples displayed a higher similarity of bacterial community than other intestinal sections, as evaluated by insignificant data of alpha and beta diversity. However, several significant differences of diversity indices were observed in the jejunum, colon, and cecum, particularly the obvious clusters of beta diversity between the healthy and the PEDV-infected piglets, indicating the community of the gut microbiota was markedly altered by PEDV infection. Similar findings were obtained by Huang et al. (16) and (17). Interestingly, increased alpha diversity indices (Shannon and Simpson) were observed in the PEDV-infected piglets, implying PEDV infection led to the dysbiosis of porcine microbiome. This result is similar to the finding of Huang et al. (16) that alpha diversity increased with time during PEDV infection, but is contrary to results of Song et al. (12) and (17). Nevertheless, Chao index in the ileum was found as the only difference between the control and NAC groups, no other variation was found between the control and NAC groups and between the PEDV and the PEDV+NAC groups, suggesting a slight effect of NAC on the bacterial community.

Characterization of bacterial composition in this research demonstrated remarkable variations caused by PEDV infection. Profound shifts in the microbiota composition at the phylum level were found in the jejunum, colon, and cecum. Particularly, the PEDV and the PEDV+NAC groups displayed a lower abundance of Bacteroidetes and a higher abundance of Proteobacteria, Firmicutes, and Fusobacteria than the control and the NAC groups in the colon and cecum. The phylum Bacteroides is important for carbohydrate fermentation and polysaccharide catabolism, as well as amino acid and protein utilization (27). The phylum Firmicutes has been shown to be involved in energy resorption, and potentially related to the development of obesity and inflammation (28). Many studies suggested that Proteobacteria and Fusobacteria are associated with the imbalance of microbiota, inflammations, and various clinical anaerobic infections $(29,30)$. These results suggest that PEDV infection caused severe alterations in the composition of the gut microbiota in piglets, which could lead to the dysbiosis and affect intestinal metabolism and host health. However, similar microbiota composition was observed between the control and the NAC groups as well as between the PEDV and the PEDV+NAC groups, implying a limited effect of NAC supplementation on the composition of bacterial community in either the healthy or the PEDV-infected piglets.

The abundance of several OTUs showed significant differences between the healthy and the PEDV infected piglets. Interestingly, PEDV infection was associated with decreased proportions of Shigella and increased proportions of Lactobacillus, Odoribacter, Anaerovibrio, Helicobacter, Lachnospiraceae_g and Sutterella. Shigella species generally invade the epithelial lining of the colon, causing severe inflammation and death of the cells lining the colon, which could result in the diarrhea and even dysentery $(31,32)$. However, the reduction of Shigella in this study was only observed in the jejunum, which may be ascribed to the frequent flow of intestinal content induced by the diarrhea. Genera Helicobacter and Sutterella are typical pathogenic bacteria, which have been frequently associated with human diseases, such as stomach cancer and inflammatory bowel disease $(33,34)$. Increased Helicobacter and Sutterella abundances suggested the adverse healthy effect of PEDV infection. Lactobacillus is commonly investigated as probiotic agents, which influences host immunity and disease susceptibility (35). Increased Lactobacillus was also observed in PEDVinfected piglets (16), which was hypothesized to be the effect of pathogen inhibition or immunomodulation of the host. The genus Odoribacter and the family Lachnospiraceae are SCFAsproducing bacteria $(36,37)$, Anaerovibrio is often associated with lipolysis (38). The increase of these metabolic-related bacteria may involve in metabolic regulations of the host, or metabolic disorder induced by the dysbiosis during PEDV infection. Future investigation is warranted to unravel the complex relationship between the microbiome shifts and PEDV infection.

In this study, NAC supplementation presented different trends in the alteration of gut microbiome in the healthy piglets vs. the PEDV-infected piglets. In the healthy piglets, NAC supplementation decreased the proportion of family Rikenellaceae and increased the proportion of genera Streptococcus and Enterococcus; while in the PEDV-infected piglets, NAC was associated with decreased proportions of Oscillospira and Prevotella. Previous evidence showed that the members of family Rikenellaceae showed a high potential for fermenting dietary proteins (39), and enriched in mice fed a high-fat diet (40). Streptococcus populations are primary fermenters of diet-derived simple sugars in the small intestine (41), as evaluated by the high expression of genes involved in primary carbohydrate transport systems by the small intestinal Streptococcus (42). Decreased Rikenellaceae and increased Streptococcus by NAC supplementation indicated that NAC played a positive role in the metabolic regulation of the healthy piglets. Moreover, Enterococcus spp. have been reported to produce bacteriocins which inhibit Gram-positive food-borne bacteria and intestinal pathogens $(43,44)$, increased Enterococcus by NAC supplementation implied a potential to improve immunity of the healthy piglets. Recent research reported that Oscillospira is often associated with leanness (45), while Prevotella was negatively associated with the severity of diarrhea-predominant irritable bowel syndrome $(46,47)$. NAC supplementation decreased the abundance of Oscillospira and Prevotella in the PEDV-infected piglets, implying that NAC 
could promote the growth of piglets and mitigate diarrhea during PEDV infection. Additionally, NAC supplementation increased the abundance of Lactobacillus in both the healthy and the PEDV-infected piglets, suggesting a beneficial effect of NAC on the regulation of the gut microbiota in the suckling piglets.

A few studies have revealed changes in the functional potential of microbiome in relation to PEDV infection, which was found to attenuate metabolism and defense mechanisms and enhance transcription and membrane transport (15-17). Similar results were obtained in the present study, reflecting that attenuating metabolism, barrier, and immune is the major functional shift of microbiome during PEDV infection. However, in the PEDVinfected piglets, many functional potentials of the microbiome were remarkably influenced by NAC supplementation. For instance, decreased amino acid metabolism, cell signaling, cellular community, multiple disease-related pathways, and endocrine and excretory system in the cecum. These results demonstrated that functional changes of the gut microbiome may be a way that NAC protects the piglets from PEDV infection.

\section{CONCLUSION}

This study evaluated the effect of dietary supplementation with NAC on the gut microbiome in the healthy and PEDVinfected piglets. PEDV infection caused severe dysbiosis of gut microbiome, as demonstrated by the community variation (beta diversity) and the composition shifts (decreased Shigella and increased Lactobacillus, Odoribacter, Anaerovibrio, Helicobacter, Lachnospiraceae_g, and Sutterella), resulted in functional changes associated with metabolism, barrier and immune. However, NAC supplementation played a positive role in regulating the gut microbiome during PEDV infection, as indicated by the altered bacterial populations (decreased Oscillospira and Prevotella and increased Lactobacillus) and function potentials (amino acid metabolism, cell signaling, cellular community, diseases,

\section{REFERENCES}

1. Jandhyala SM, Talukdar R, Subramanyam C, Vuyyuru H, Sasikala M, Reddy DN. Role of the normal gut microbiota. World J Gastroenterol. (2015) 21:8787. doi: 10.3748/wjg.v21.i29.8787

2. Inta D, Wolnerhanssen BK, Meyer-Gerspach AC, Lang E, Schweinfurth N, Mallien AS, et al. Common pathways in depression and obesity: the role of gut microbiome and diets. Curr Behav Neurosci Rep. (2020) 7:15-21. doi: 10.1007/s40473-020-00199-1

3. Baothman OA, Zamzami MA, Taher I, Abubaker J, Abu-Farha M. The role of gut microbiota in the development of obesity and diabetes. Lipids Health Dis. (2016) 15:108. doi: 10.1186/s12944-016-0278-4

4. Andoh A. Physiological role of gut microbiota for maintaining human health. Digestion. (2016) 93:176-81. doi: 10.1159/000444066

5. Ni J, Wu GD, Albenberg L, Tomov VT. Gut microbiota and IBD: causation or correlation? Nat Rev Gastro Hepat. (2017) 14:573. doi: 10.1038/nrgastro.2017.88

6. Pickard JM, Zeng MY, Caruso R, Nunez G. Gut microbiota: role in pathogen colonization, immune responses, and inflammatory disease. Immunol Rev. (2017) 279:70-89. doi: 10.1111/imr.12567

7. Feng Q, Chen WD, Wang YD. Gut microbiota: an integral moderator in health and disease. Front Microbiol. (2018) 9:151. doi: 10.3389/fmicb.2018.00151 endocrine, and excretory system). Therefore, regulating the gut microbiome by anti-PEDV(antiviral) substances or compounds could be taken as a promising candidate for the prevention or treatment of PEDV infection.

\section{DATA AVAILABILITY STATEMENT}

The original contributions presented in the study are deposited in the Majorbio Cloud Platform database (www.majorbio.com). The datasets generated for this study are available on request to the corresponding author.

\section{ETHICS STATEMENT}

The animal study was reviewed and approved by Animal Care and Use Committee of Wuhan Polytechnic University.

\section{AUTHOR CONTRIBUTIONS}

TW and YH planned the project and designed the experiments. TW, YL, and XL conducted the experiments. YL carried out the data analysis. MW, KY, SL, and CJ contributed the animal trials. $\mathrm{XL}$ and $\mathrm{DZ}$ contributed reagents preparation and samples collection. YL and TW wrote the manuscript and reviewed by QZ, YZ, and DY. All authors have read and agreed to the published version of the manuscript.

\section{FUNDING}

This research was supported by the National Key R\&D Program of China (2017YFD0500505), Open Project of Hubei Key Laboratory of Animal Nutrition and Feed Science (Grant Number 201905), and Hubei Provincial Technology and Innovation Program (2017AHB062).
8. Ramos CP, Santana JA, Morcatti Coura F, Xavier RGC, Leal CAG, Oliveira Junior CA, et al. Identification and characterization of Escherichia coli, salmOnella spp., Clostridium perfringens, and C. difficile isolates from reptiles in Brazil. BioMed Res Int. (2019) 2019:9530732. doi: 10.1155/2019/9530732

9. Lee C. Porcine epidemic diarrhea virus: an emerging and re-emerging epizootic swine virus. Virol J. (2015) 12:193. doi: 10.1186/s12985-015-0421-2

10. Carvajal A, Arguello H, Martínez-Lobo FJ, Costillas S, Miranda R, de nova PJ, et al. Porcine epidemic diarrhoea: new insights into an old disease. Porc Health Manag. (2015) 1:12. doi: 10.1186/s40813-015-0007-9

11. Gallien S, Moro A, Lediguerher G, Catinot V, Paboeuf F, Bigault L, et al. Evidence of porcine epidemic diarrhea virus (PEDV) shedding in semen from infected specific pathogen-free boars. Vet Res. (2018) 49:7. doi: 10.1186/s13567-018-0505-2

12. Song D, Peng Q, Chen Y, Zhou X, Zhang F, Li A, et al. Altered gut microbiota profiles in sows and neonatal piglets associated with porcine epidemic diarrhea virus infection. Sci Rep. (2017) 7:1-10. doi: 10.1038/s41598-017-17830-z

13. Koh HW, Kim MS, Lee JS, Kim H, Park SJ. Changes in the swine gut microbiota in response to porcine epidemic diarrhea infection. Microbes Environ. (2015) 30:284-7. doi: 10.1264/jsme2.ME15046

14. Liu S, Zhao L, Zhai Z, Zhao W, Ding J, Dai R, et al. Porcine epidemic diarrhea virus infection induced the unbalance of gut microbiota 
in piglets. Curr Microbiol. (2015) 71:643-9. doi: 10.1007/s00284-015-0 895-6

15. Huang MZ, Wang SY, Wang H, Cui DA, Yang YJ, Liu XW, et al. Differences in the intestinal microbiota between uninfected piglets and piglets infected with porcine epidemic diarrhea virus. PLoS ONE. (2018) 13:e0192992. doi: 10.1371/journal.pone.0192992

16. Huang A, Cai R, Wang Q, Shi L, Li C, Yan H. Dynamic change of gut microbiota during porcine epidemic diarrhea virus infection in suckling piglets. Front Microbiol. (2019) 10:322. doi: 10.3389/fmicb.2019.00322

17. Tan Z, Dong W, Ding Y, Ding X, Zhang Q, Jiang L. Porcine epidemic diarrhea altered colonic microbiota communities in suckling piglets. Genes. (2020) 11:44. doi: 10.3390/genes11010044

18. Hou YQ, Wang L, Yi D, Ding BY, Yang ZG, Li J, et al. N-acetylcysteine reduces inflammation in the small intestine by regulating redox, EGF and TLR4 signaling. Amino Acids. (2013) 45:513-22. doi: 10.1007/s00726-012-1295-x

19. Hou YQ, Wang L, Zhang W, Yang ZG, Ding BY, Zhu HL, et al. Protective effects of $\mathrm{N}$-acetylcysteine on intestinal functions of piglets challenged with lipopolysaccharide. Amino Acids. (2012) 43:1233-42. doi: 10.1007/s00726-011-1191-9

20. Wu G. Dietary requirements of synthesizable amino acids by animals: a paradigm shift in protein nutrition. J Anim Sci Biotechnol. (2014) 5:34. doi: 10.1186/2049-1891-5-34

21. Schairer DO, Chouake JS, Kutner AJ, Makdisi J, Nosanchuk JD, Friedman AJ. Evaluation of the antibiotic properties of glutathione. J Drugs Dermatol. (2013) 12:1272-7. doi: 10.1016/j.jaad.2014.01.131

22. Goswami M, Jawali N. N-acetylcysteine-mediated modulation of bacterial antibiotic susceptibility. Antimicrob Agents Chemother. (2010) 54:3529-30. doi: 10.1128/AAC.00710-10

23. Samuni Y, Goldstein S, Dean OM, Berk M. The chemistry and biological activities of N-acetylcysteine. Biochim Biophys Acta. (2013) 1830:4117-29. doi: 10.1016/j.bbagen.2013.04.016

24. Du J, Luo J, Yu J, Mao X, Luo Y, Zheng P, et al. Manipulation of intestinal antiviral innate immunity and immune evasion strategies of porcine epidemic diarrhea virus. BioMed Res. Int. (2019) 2019:e1862531. doi: $10.1155 / 2019 / 1862531$

25. Wang L, Zhou J, Hou Y, Yi D, Ding B, Xie J, et al. N-Acetylcysteine supplementation alleviates intestinal injury in piglets infected by porcine epidemic diarrhea virus. Amino Acids. (2017) 49:1931-43. doi: 10.1007/s00726-017-2397-2

26. Fu S, Zhuang F, Guo L, Qiu Y, Xiong J, Ye C, et al. Effect of baicalin-aluminum complexes on fecal microbiome in piglets. Int J Mol Sci. (2019) 20:2390. doi: 10.3390/ijms20102390

27. Hooper LV, Midtvedt T, Gordon JI. How host-microbial interactions shape the nutrient environment of the mammalian intestine. Annu Rev Nutr. (2002) 22:283-307. doi: 10.1146/annurev.nutr.22.011602.092259

28. Ley RE, Turnbaugh PJ, Klein S, Gordon JI. Human gut microbes associated with obesity. Nature. (2006) 444:1022-3. doi: 10.1038/4441022a

29. Swidsinski A, Dorel Y, Loening-Baucke V, Theissig F, Ruckert JC, Ismail $\mathrm{M}$, et al. Acute appendicitis is characterised by local invasion with fusobacterium nucleatum/necrophorum. Gut. (2011) 60:34-40. doi: 10.1136/gut.2009.191320

30. Bennett JE, Dolin R, Blaser MJ. Mandell, Douglas, and Bennett's Principles and Practice of Infectious Diseases. Vol. 2. New York, NY: Elsevier Health Sciences (2014).

31. Kotloff KL, Nataro JP, Blackwelder WC, Nasrin D, Farag TH, Panchalingam $\mathrm{S}$, et al. Burden and aetiology of diarrhoeal disease in infants and young children in developing countries (the Global Enteric Multicenter Study, GEMS): a prospective, case-control study. Lancet. (2013) 382:209-22. doi: 10.1016/S0140-6736(13)60844-2

32. Mani S, Wierzba T, Walker RI. Status of vaccine research and development for Shigella. Vaccine. (2016) 34:2887-94. doi: 10.1016/j.vaccine.2016.02.075

33. Hua JS, Zheng PY, Ho B. Species differentiation and identification in the genus of helicobacter. World J Gastroenterol. (1999) 5:7-9. doi: 10.3748/wjg.v5.i1.7
34. Hiippala K, Kainulainen V, Kalliomaki M, Arkkila P, Satokari R. Mucosal prevalence and interactions with the epithelium indicate commensalism of Sutterella spp. Front Microbiol. (2016) 7:1706. doi: 10.3389/fmicb.2016.01706

35. Niederwerder MC. Role of the microbiome in swine respiratory disease. Vet Microbiol. (2017) 209:97-106. doi: 10.1016/j.vetmic.2017.02.017

36. Gomez-Arango LF, Barrett H, McIntyre D, Callaway LK, Morrison $\mathrm{M}$, Nitert MD. Increased systolic and diastolic blood pressure is associated with altered gut microbiota composition and butyrate production in early pregnancy. Hypertension. (2016) 68:974-7. doi: 10.1161/HYPERTENSIONAHA.116.07910

37. Boutard M, Cerisy T. Functional diversity of carbohydrate-active enzymes enabling a bacterium to ferment plant biomass. PLoS Genet. (2014) 10:e1004773. doi: 10.1371/journal.pgen.1004773

38. Shingfield KJ, Wallace RJ. CHAPTER 1:synthesis of conjugated linoleic acid in ruminants and humans, in conjugated linoleic acids and conjugated vegetable oils. Roy Soc Chem. (2014) 1-65. doi: 10.1039/9781782620211-00001

39. Su XL, Tian Q, Zhang J, Yuan XZ, Shi XS, Guo RB, et al. Acetobacteroides hydrogenigenes gen. nov., sp. nov., an anaerobic hydrogen-producing bacterium in the family Rikenellaceae isolated from a reed swamp. Int J Syst Evol Microbiol. (2014) 64:2986-91. doi: 10.1099/ijs.0.063917-0

40. Kim KA, Gu W, Lee IA, Joh EH, Kim DH. High fat diet-induced gut microbiota exacerbates inflammation and obesity in mice via the TLR4 signaling pathway. PLoS ONE. (2012) 7:e47713. doi: 10.1371/journal.pone.0047713

41. van den Bogert B, Meijerink M, Zoetendal EG, Wells JM, Kleerebezem M. Immunomodulatory properties of Streptococcus and veillonella isolates from the human small intestine microbiota. PLoS ONE. (2014) 9:e114277. doi: 10.1371/journal.pone.0114277

42. Zoetendal EG, Raes J, van den Bogert B, Arumugam M, Booijink CC, Troost FJ, et al. The human small intestinal microbiota is driven by rapid uptake and conversion of simple carbohydrates. ISME J. (2012) 6:1415-26. doi: 10.1038/ismej.2011.212

43. Franz CMAP, Worobo RW, Quadri LE, Schillinger U, Holzapfel WH, Vederas JC, et al. Atypical genetic locus associated with constitutive production of enterocin B by Enterococcus faecium BFE 900. Appl Environ Microbiol. (1999) 65:2170-8. doi: 10.1128/AEM.65.5.2170-2178.1999

44. Toit MD, Franz CMAP, Dicks LMT, Holzapfel WH. Preliminary characterization of bacteriocins produced by Enterococcus faecium and Enterococcus faecalis isolated from pig faeces. J Appl Microbiol. (2000) 88:482-94. doi: 10.1046/j.1365-2672.2000.00986.x

45. Gophna U, Konikoff T, Nielsen HB. Oscillospira and related bacteria-From metagenomic species to metabolic features. Environ Microbiol. (2017) 19:83541. doi: 10.1111/1462-2920.13658

46. Tap J, Derrien M, Tornblom H, Brazeilles R, Cools-Portier S, Dore J, et al. Identification of an intestinal microbiota signature associated with severity of irritable bowel syndrome. Gastroenterology. (2017) 152:111-23. doi: 10.1053/j.gastro.2016.09.049

47. Su T, Liu R, Lee A, Long Y, Du L, Lai S, et al. Altered intestinal microbiota with increased abundance of prevotella is associated with high risk of diarrhea-predominant irritable bowel syndrome. Gastroenterol Res Pract. (2018) 2018:e6961783. doi: 10.1155/2018/6961783

Conflict of Interest: The authors declare that the research was conducted in the absence of any commercial or financial relationships that could be construed as a potential conflict of interest.

Copyright (c) $2021 \mathrm{Wu}, \mathrm{Lyu}, \mathrm{Li}, \mathrm{Wu}, \mathrm{Yu}, \mathrm{Li}$, Ji, Zhang, Zhang, Zhao, Yi and Hou. This is an open-access article distributed under the terms of the Creative Commons Attribution License (CC BY). The use, distribution or reproduction in other forums is permitted, provided the original author(s) and the copyright owner(s) are credited and that the original publication in this journal is cited, in accordance with accepted academic practice. No use, distribution or reproduction is permitted which does not comply with these terms. 